PACS 32.80.Rm, 05.45.+b;

УДК 535.42.,539.184.

\title{
LASER PHOTOIONIZATION SENSOR OF THE SEPARATING ISOTOPES AND NUCLEAR REACTIONS PRODUCTS: THEORETICAL FOUNDATIONS OF NEW SCHEME
}

\author{
S. V. Ambrosov \\ Institute of Applied mathematics OSEU, P.O.Box 108, Odessa-9, 65009, Ukraine \\ Phone: +380-482-637227 E-mail: glushkov@paco.net
}

\section{Summary \\ LASER PHOTOIONIZATION SENSOR OF THE SEPARATING ISOTOPES \\ AND NUCLEAR REACTIONS PRODUCTS: THEORETICAL FOUNDATIONS OF NEW SCHEME}

S. V. Ambrosov

It is proposed and studied a new optimal theoretical scheme for the laser photoionization sensor of the separating isotopes, nuclear isomers and nuclear reactions products, which is based on the selective laser excitation of the isotope atoms into excited Rydberg states and further stochastic collisional ionization mechanism. The operator perturbation theory and FockerPlank stochastic equation method are used for description of stochastic collisional ionization process. The optimal laser action model and density matrices formalism is used for numeric calculation of the optimal scheme of the $N a, U, C s$ and $C f$ isotopes (ions, nuclei) sensing.

Key words: laser photoionization sensor, nuclear reaction products, isotoes

\section{Анотація}

\section{ЛАЗЕРНИЙ ФОТОІОНІЗАЦІЙНИЙ СЕНСОР ІЗОТОПІВ \\ ТА ПРОДУКТІВ ЯДЕРНИХ РЕАКЦІЙ: ТЕОРЕТИЧНІ ОСНОВИ НОВОЇ СХЕМИ}

\section{С. В. Амбросов}

Вперше запропоновано лазерний фотоіонізаційний сенсор ізотопів, ядерних ізомерів та продуктів ядерних реакцій. В основі його реалізації лежить отимальна теоретична схема селективного лазерного збудження атомів ізотопів у рідбергівські стани та механізм подальшої стохастичної іонізації за рахунок зіткнень. Операторна теорія збурень та метод стохастичного рівняння Фоккера-Планка використано для опису процесу стохастичної іоні-зації за рахунок зіткнень. Модель оптимальних лазерних діянь і апарат матриці густини за-стосовано для чисельного моделювання оптимальної схеми детектування ізотопів $N a, U, C s C f$

Ключові слова: лазерний фотоіонізаційний сенсор, продукти ядерної реакції, ізотопи 


\title{
Аннотация \\ ЛАЗЕРНЫЙ ФОТОИОНИЗАЦИОННЫЙ СЕНСОР ИЗОТОПОВ И ПРОДУКТОВ ЯДЕРНЫХ РЕАКЦИЙ: ТЕОРЕТИЧЕСКИЕ ОСНОВЫ НОВОЙ СХЕМЫ
}

\author{
С. В. Амбросов
}

Впервые предложен лазерный фото ионизационный сенсор изотопов, ядерных изомеров и продуктов ядерных реакций. В основе его реализации лежат оптимальная теоретическая схема селективного лазерного фото возбуждения атомов в ридберговские состояния и механизме дальнейшей стохастической столкновительной ионизации. Операторная теория возмущений и метод стохастических уравнений применены для описания процесса стохастической столкновительной ионизаци. Модель оптимальных лазерных воздействий и аппарат матрицы плотности использованы для численного моделирования оптимальной схемы детектирования изотопов $N a, U, C s$ и $C f$.

Ключевые слова: лазерный фотоионизационный сенсор, продуктов ядерной реакции, изотопы

\section{Introduction}

One of the most perspective methods for the sensing single atoms, separating isotopes, nuclear isomers and nuclear reactions products is laser photoionization method. (c.f.[1-15]). The standard laser photoionization sensor scheme may be realized on the basis of a method of the multi-step excitement and ionization of atoms by laser radiation. The method of selective photoionization of atoms, based on the selective resonance excitation of atoms by laser radiation into states near ionization boundary and further photo-ionization of the excited states by additional laser radiation, has been at first proposed by Letokhov (c.f. [1]). This method represents a great interest for laser separation of the isotopes, nuclear isomers and optically discharged gases separation. Due to the known significant disadvantage of the two-step selective ionization of atoms by laser radiation method, quite perspective photoionization scheme bases on the use of the autoionization levels. Hitherto, detailed data about autoionization resonances for many atoms are often absent. In a number of papers (c.f. $[1,9,11,151])$ a possibility of the selective ionization of atoms, based on the selective resonance excitation of atoms by laser radiation into states near ionization boundary and further ionization decay of excited atoms by external electric field, has been considered. Electric field changes the electron spectra so that the part of discrete spectra levels (near the ionization boundary) part moves into continuum and other levels become by the autoionization ones. The probability of their autoionization de- cay quickly increases with growth of the main quantum number. The most optimal situation is when atom is excited to state, which has the autoionization probability more than the radiation decay one. To receive an adequate data regarding the laser photoionization sensor optimal schemes, it is necessary to carry out accurate calculating the process of excitement of atoms by laser field and the probability of ionization of the highly excited atoms by electric field. In last years the accurate calculations of elementary atomic processes in different photoionization schemes were carried out, including the photoionization schemes with the use of the autoionization resonances [8-25]. As a rule, non-relativistic approximation has been used [9]. More consistent approach to solution of such a problem must be based on the relativistic models [8,12], as the most interesting elements for laser isotope separation are heavy and a role of relativistic corrections is often very dramatic. From another side, as alternative mechanism for ionization of excited atoms one could use an action of the collisional ionization (an action of electron impact, atom-atom or ion-atom collisions etc.) (c.f.[8,10,20-24]. It should be noted that such scheme of the laser photoionization sensor is technically naturally realized by means of the plasmotronic technique [2]. Though many of cited processes are sufficiently adequately described by simplified models, at least on qualitative level, a complete, consistent theory of these processes is absent hitherto [18-20]. In ref.[7-11] it is developed an effective approach to modelling optimal schemes for the laser photoionization sensors of isotopes and nu- 
clear isomers, which is based on the complex use of the elementary atomic processes models, optimal laser actions models, density matrices formalism. Results of numeral calculation for optimal scheme of the isotope separation for alkali elements were presented. On second step of the photoionization scheme, it was used the collisional mechanism, provided by ionization through the electron impact. This paper goes our research on carrying out optimal schemes for the laser photoionization sensors of separating isotopes, nuclear isomers and nuclear reactions products. New optimal scheme is based on the selective laser excitation of the isotope atoms into excited Rydberg states and further stochastic collisional ionization mechanism. It should be emphasized that we propose at first to use a stochastic collisional process as last step of the photoionization scheme. The matter is in a fact that the cross-section of ionization in stochastic regime is significantly higher than one in the usual regime (c.f. $[6,10,23]$ ). A result, the effectiveness of the laser photoionization sensor scheme is significantly increased. The operator perturbation theory and Focker-Plank stochastic equation method are used for description of stochastic collisional ionization process. The optimal laser action model and density matrices formalism is used for numeric calculation of the optimal scheme of the $N a, U, C s$ and $C f$ isotopes (ions, nuclei) sensing.

\section{Key steps of the laser photoionization scheme}

In practical realization of the laser photoionization schemes, as a rule, the mixture of buffer and separated gases (isotopes) moves along the electric discharge zone. The discharge zone is radiated by external electromagnetic field, which is resonant to one from chosen isotopes [1]. The buffer gas defines the discharge characteristics (temperature $\mathrm{T}$, concentration of particles $n$ ). In the discharged zone the isotopes are ionized and then are removed from the discharged zone and collected by one of the physically chemical methods. Because of the difference for diffusion coefficients, under perpendicular gas stream velocity, the ions will reach the surrounding walls more rapidly then atom with further localization due to the ionization process. The ionization time is corresponding to the time of passing for atom through the electromagnetic field action zone and equal: $\tau=t_{f}=L / v$ ( L- size of radiated zone; $\mathrm{v}$ - velocity of particles stream). If a resonant transition is satu- rated by a laser radiation and it is realized a collisional mechanism of ionization for excited atoms then a condition for ionization of the resonant component is as follows: $1 / n S^{\prime}(T)>1 / v=t_{f}$ (here $S^{\prime}$ - coefficient of ionization of excited atoms in collision). The typical values for indicated parameters are following: $L=1 \mathrm{sm}, v=10^{4} \mathrm{sm} / \mathrm{s}, n=\left(10^{11}-10^{13}\right) \mathrm{cm}^{-3}$. As the collisional ionization mechanism is further considered, it is useful to make substitutions: $W_{2} \rightarrow \mathrm{S}^{\prime} \mathrm{n} / \gamma$, $\tau \rightarrow \gamma z / v, E_{f}=\sigma_{12} w_{d} / v d \hbar w_{21}$ (here z- space co-ordinate along stream; $d$ - transverse sizes of radiated zone). The typical values are as follows: $S^{\prime} n=10^{4} \mathrm{~s}^{-1}$, $\gamma=10^{4} \mathrm{~s}^{-1}, t_{f}=4 \cdot 10^{-4} \mathrm{~s}, E_{f}=2,5$. Consider in details the selective ionization scheme for $\mathrm{Na}$ ( $\mathrm{Na}$ vapours at temperature $600 \mathrm{~K})$. The first ionization step: excitation of $\mathrm{Na}$ atoms to state $3 p^{2} P_{1 / 2}$ and then photoionization by laser pulse: the excitation cross-section $\sigma_{I}=10^{-12}$ $10^{-11} \mathrm{~cm}^{2}$, ionization cross-section from excited state: $\sigma_{2}=10^{-17} \mathrm{~cm}^{2}$, from ground state $\sigma_{2}=10^{-19} \mathrm{~cm}^{2}[1]$. The experimental studying the of the $\mathrm{Na}$ vapours in laser field was fulfilled by Letokhov V.S. et al [1]. For selective photoionization scheme with excitation to Rydberg $S, P, D$ states with $n=10-18$ and further ionization by electric field $(E=10 \mathrm{KV} / \mathrm{cm})$ calculation $[9,13]$ (cross-section is defined in standard way) has given the values: for the $3 p^{2} P_{1 / 2}-15^{2} D_{3 / 2}$ transition $\sigma_{2}=7,1 \cdot 10^{-15} \mathrm{~cm}^{2}$, theoretical estimate of ref. [8] $\sigma_{2}=7,6 \cdot 10^{-15} \mathrm{~cm}^{2}$, experimental value: $\sigma_{2}=7 \cdot 10^{-15} \mathrm{~cm}^{2}$. So, a selective photoionization scheme with using the Rydberg states and ionization by external electric field is quite effective for alkali elements from the energetic point of view, but the ionization output here is less than $100 \%$ ! This is one of the most disadvantages of the scheme with ionization by an external electric field for non-alkali elements (lanthanide's, actinides etc.). As it was indicated earlier, an alternative mechanism is the collisional ionization. It is stressed that hitherto this ionization mechanism is not sufficiently studied for solution of the isotopes separation laser photoionization problem and technological realization of the laser photoionization sensor. The typical ionization processes are as follows [21]:

$$
\begin{gathered}
A^{*}(n l)+B \rightarrow\left(A+B^{+}\right)+e \text { or } \\
A^{*}(n l)+B \rightarrow\left(A^{+}+B\right)+e \text { or } \\
A^{*}(n l)+B \rightarrow A B^{+}+e .
\end{gathered}
$$

Here $\mathrm{A}^{*}$ denotes an atom in an excited state, $\mathrm{B}^{+}-$ an ionized atom, other notations are obvious. The process (1c) is called as an associative ionization. As a rule, it realizes effectively when dissociation ener- 
gy of molecular ion $A B^{+}$is higher than ionization potential of excited atom. In application to carrying out the isotopes separation laser photoionization scheme this process has been considered in a number of papers (c.f. ref. [1]). Its optimal variant is at first proposed in ref. [10]. The first process (1a) realizes effectively when the excitation energy of atom $\mathrm{A}$ is more than the ionization potential of atom $\mathrm{B}$. If an atom $\mathrm{A}$ is in the metastable state then the process is called as the Penning ionization one. It is very curious that hitherto a consistent, highly exact, quantum mechanical approach to calculating main characteristics of this process is absent though many different simplified models has been proposed (c.f. [18-23]). The most widespread approach is based on the calculation of the capture cross-section of collisional particles by field of the van-der-Waalse interaction. Such an approach does not factually define any difference between the Penning process and resonant collisional one and gives often non-correct results for cross-sections. More consistent method requires data about the process probability $G(R)$ as function of inter nuclear distance. It should be noted that these data are practically absent at present time. Further we consider a new, consistent, stochastic model for calculation of process (1a). We believe that such mechanism may be very effective for using in the optimized laser photoionization scheme of the isotopes sensing.

\section{New stochastic collisional ionization model}

A complete cross section of the collisional process (1a) is defined by a general expression:

$$
\sigma=\int_{0}^{\infty} 2 \pi \rho d \rho\left\{1-\exp \left[-\int_{-\infty}^{+\infty} G(R) d t\right]\right\}
$$

Here $G(R)$ is a probability of the Auger effect and defined by usual way as: $G(R)=2 \pi\left|V_{12}\right|^{2} g_{2}$ (indexes 1 and 2 are relating to states: $A *+B$ and $A+B^{+}+e ; g$ is a density of the final states; $V$ is operator of interaction between atoms). In a case if the ionization process is realized in the repulsive potential of interaction between atoms in the initial channel one would write the next expression insist of formulae (2):

$$
\sigma=\left(4 \pi f_{w} / v\right) \int_{R_{m}}^{\infty} R^{2} G(R) \sqrt{1-U(R) / E} d R
$$

Here $f_{w}$ is a probability that the process is permitted on entire electron spin of system of the collision- al atoms, $v$ is the relative velocity of collision, $R_{t n}$ is the minimally possible distance of rapprochement (the turning point). To obtain correct expression for autoionization quasi-molecular width it should be used the operator perturbation theory (c.f.[9,22,25]). Within it we have taken into account a possibility of decay in the second and higher orders of perturbation theory on $V(R)$. Such approach may be used as for the Penning ionization description (asymptotic version is used in ref. [22]) as for ionization through the wan-der-Waalse capture [24]. In the perturbation theory second and higher orders it is necessary to make substitution of matrix element: $\left\langle 1\left|V(R) G_{E_{\infty}} V(R) \ldots V(R)\right| 2\right\rangle$ insist of the simple matrix element $\langle 1|V(R)| 2\rangle$ in expression for probability of collisional decay. Here $\left[1>\equiv\left[\mathrm{A}^{*}+\mathrm{B}>\right.\right.$ is the initial state, $\left[2>\equiv\left[\mathrm{A}+\mathrm{B}^{+}+\mathrm{e}>\right.\right.$ is the final state; $G_{E}$ is the Green function (see below); $E$ ? is an energy of quasi-molecule $\mathrm{A}^{*} \mathrm{~B}$ under $R \rightarrow \infty$ (the non-interacting atoms approximation). For operator $V(\mathrm{R})$ it is useful to use expansion on non-reducible tensor operators:

$$
\begin{gathered}
V(R)=\sum_{l_{1}, l_{2}=1}^{\infty} V_{l_{1} l_{2}}(n) / R^{l_{1}+l_{2}+1} \\
V_{l_{1} l_{2}}(n)=(-1)^{l_{2}} \sqrt{\frac{\left(2 l_{1}+2 l_{2}\right) !}{\left(2 l_{1}\right) !\left(2 l_{2}\right) !}}\left(C_{l_{1}+l_{2}}(n)\left\{Q_{l_{1}}^{A} \otimes Q_{l_{2}}^{B}\right\}\right), \\
\mathrm{n}=\frac{R}{R}
\end{gathered}
$$

Here $C_{l m}(\mathrm{n})$ is the modified spherical function, $\widehat{Q}_{l m}$ is an operator of the $2^{1}$-pole moment of atom.

Let us suppose that atom $\mathrm{A}^{*}$ is in a state with entire moment $J_{i}$ and projection on the quantization axe $M_{\mathrm{i}}$; in the final state the corresponding quantum numbers are $J_{f} M_{f}$; The differential (on direction $\hat{k}$ ejection of the Penning electron) probability of the $2 l_{1}$-pole- $2 l_{2}$-pole decay in the second perturbation theory order is as follows:

$$
\frac{d G(R)}{d \Omega_{k}}=\left.\frac{\left(2 l_{1}+1\right)\left(l_{1}+1\right)\left(2 l_{1}+3\right)\left(2 l_{2}+1\right)\left(l_{2}+1\right)\left(2 l_{2}+3\right)}{2 R^{2 l_{1}+2 l_{2}+8}\left[1+\delta_{l_{1} l_{2}}\right]^{2}\left(2 J_{f}+1\right)}\right|_{p_{1} p_{2} p_{3}}
$$

$$
\begin{aligned}
& \sum_{\lambda_{1} \lambda_{2} \lambda_{3}} C_{p_{3} \lambda_{3} p_{2} \lambda_{2}}^{p_{1} \lambda_{1}} \times C_{l_{2}+10 l_{1}+10}^{p_{1} 0} C_{J_{i} M_{i} p_{3} \lambda_{3}}^{J_{f} M_{f}} C_{p_{1} \lambda_{1}}^{*}(n) \times \\
& \times \sum_{J l f^{m} f}\left(2 p_{2}+1\right)\left(2 p_{3}+1\right) \exp \left(i\left(\delta_{l f}-\frac{\pi l_{f}}{2}\right)\right) \times
\end{aligned}
$$




$$
\begin{aligned}
& \times C_{l f m f}(\hat{k}) C_{l_{i} m_{i} p_{2} \lambda_{2}}^{l f m f}\left\{\begin{array}{lll}
1 & 1 & p_{2} \\
l_{f} & l_{i} & l
\end{array}\right\} \times \\
& \times\left\{\begin{array}{ccc}
1 & l_{1} & l_{1}+1 \\
1 & l_{2} & l_{2}+1 \\
p_{2} & p_{3} & p_{1}
\end{array}\right\}\left[\begin{array}{ccc}
l_{2} & l_{1} & p_{3} \\
J_{f} & J_{i} & J
\end{array}\right\} \Re_{J l}^{l f}\left(l_{1} l_{2}\right)+ \\
& \left.+(-1)^{l_{1}+l_{2}+p_{2}+p_{3}}\left\{\begin{array}{lll}
l_{1} & l_{2} & p_{3} \\
J f & J i & J
\end{array}\right\} \Re_{J l}^{l f}\left(l_{2}, l_{1}\right)\right]\left.\right|^{2}
\end{aligned}
$$

Here the reducible matrix elements are represented as:

$$
\Re_{J l}^{l f}\left(l_{2}, l_{1}\right)=<n_{A} J_{i} ; O_{B} l_{i}\left\|\widehat{Q}_{l_{2}}^{A} \hat{d}^{B} g_{J}^{A^{*} B_{0}} \widehat{Q}_{l_{1}^{A}}^{A} \widehat{d}^{B}\right\| O_{A} J_{f} ; E l_{f}>
$$

Here $d^{B}=Q^{\mathrm{B}}$ is an operator of the dipole moment of atom $B, g^{A * B O}$-is a radial Green function. Because of that the final state of atom $B \mid E 1_{f}>$ is a state of continuum with scattering phase $\delta_{l f}$ then the fine structure of levels in atom B may not be accounted. To calculate (6) we used the model perturbation theory with pseudopotential zeroth approximation and effective account of the exchange-correlation effects [23-25] and complex of molecular programs "Superstructure" [9-17]. In this aspect our approach differs from the analogous approach of Manakov et al [21]. The final expression for entire probability of the electron ejection is

$$
\begin{gathered}
G(R)=\left.\frac{2 \pi\left(2 l_{1}+1\right)\left(l_{1}+1\right)\left(2 l_{1}+3\right)\left(2 l_{2}+1\right)\left(l_{2}+1\right)\left(2 l_{2}+3\right)}{R^{2 l_{1}+2 l_{2}+8}\left(2 l_{i}+1\right)\left[1+\delta_{l_{1} l_{2}}\right]^{2}\left(2 J_{i}+1\right)}\right|_{p_{1} p_{2} p_{3}} \\
\sum_{l_{f}}\left(C_{l_{2}+10 l_{1}+10}^{p_{1} 0}\right)^{2} \times
\end{gathered}
$$$$
\times\left(2 p_{2}+1\right)\left(2 p_{3}+1\right)\left\{\begin{array}{ccc}
1 & l_{1} & l_{1}+1 \\
1 & l_{2} & l_{2}+1 \\
p_{2} & p_{3} & p_{1}
\end{array}\right\} \mid \sum_{l l}\left\{\begin{array}{ccc}
1 & 1 & p_{2} \\
l_{f} & l_{i} & l
\end{array}\right\} \times
$$$$
\times\left[\begin{array}{ccc}
l_{2} & l_{1} & p_{3} \\
J_{f} & J_{i} & J
\end{array}\right\} \Re_{J l}^{l f}\left(l_{1} l_{2}\right)+
$$$$
\left.+(-1)^{l_{1}+l_{2}+p_{2}+p_{3}}\left\{\begin{array}{lll}
l_{1} & l_{2} & p_{3} \\
J f & J i & J
\end{array}\right\} \Re_{J l}^{l f}\left(l_{2}, l_{1}\right)\right]\left.\right|^{2} .
$$

The key moment of calculation is connected with calculation of the diatomic radial matrix elements. Technical difficulties of such calculation are known. The effective procedure for two-times summation on the entire set of the collisional atoms states is earlier developed (c.f. ref. $[9,14,23])$.
In a case of participating highly excited atom A in processes (1a) one could suppose a possibility of realization of the stochastic mechanism for ionization. From physical point of view it is provided by a chaotic drift of the Rydberg electron, which interacts with the electromagnetic field of dipole. So, it is possible to use the theory of chaotic drift of the Coulomb electron in the microwave fields (c.f. $[9,18,22])$. One can introduce the function of distribution $f(n, t)$ of the Rydberg electron on space of effective quantum numbers $n$. The equation of motion of the Rydberg electron in our case can be written as:

$$
\begin{aligned}
\partial f(n, t) / \partial t & =\partial / \partial n\left[\Theta\left(n-N_{\min }\right) D(R) n^{3} \partial f(n, t) / \partial n\right]- \\
& -\Theta\left(n-N_{\max }\right) G(n, R) f(n, t)
\end{aligned}
$$

Here $\Theta\left(n-N_{\text {min }}\right)$ is the Heviside function as an additive multiplier in the coefficient of diffusion $D(R) n^{3}$, which provides "freezing" the stochastic processes in region of the low lying states in accordance with the known Cirikov criterion. This criterion defines the region of stochasticity under condition: $N_{\min }<n<N_{\max }$. In the region of highly excited states $\left(n>N_{\max }\right)$ it is opened a direct channel of ionization and the electron is ejected. The average effective time $\tau_{d i f}$ for diffusion of electron from level $n=n_{0}{ }^{*}$ till the ionization threshold $N_{\max }\left(R_{t n}\right)$ and further output into continuum is determined by equation:

$$
\begin{gathered}
<\tau_{d i j}>\left(n_{0}{ }^{*}\right)=1 / n_{o}{ }^{*}-1 / N_{\text {max }}\left(R_{t n}\right)++ \\
+N_{\text {min }}\left(R_{t n}\right) / 2 N^{2}{ }_{\text {max }}\left(R_{t n}\right)-N_{\text {min }}\left(R_{t n}\right) / 2\left(n_{0}{ }^{*}\right)^{2}
\end{gathered}
$$

The details of deriving and defining the coefficients are considered in ref.[22] and cited references. The effective collision time is defined from equality: $<\tau_{d i f}>\left(n_{0}{ }^{*}\right)=t_{c o l}\left(R_{t n}\right)$. The value of the turning point $R_{t n}$ is found from the last equality and equations $(4,8,9)$. The constant of ionization $K_{i}$ is calculated in accordance to definition as follows:

$$
\begin{gathered}
K_{i}=\int_{0}^{\infty} d E_{c} F\left(E_{c}\right) \sqrt{2 / \mu} \sqrt{E_{c}} \sigma_{i}\left(E_{c}\right), \\
F\left(E_{c}\right)=\left(4 \cdot E_{c}^{2} / T^{3}\right) \exp \left(-2 E_{c} / T\right)
\end{gathered}
$$

Definition of he collision function and other parameters is given in ref. [20-23]. It should be noted that naturally a model proposed can be used in calculations of probabilities for different collision processes and effects in atomic gases (not only the Penning ionization process and analogous ones). Let us consider some numerical results, obtained on the basis of calculation within above presented model of the stochastic collision ionization. The reaction (1a) 
for pair: "Atom of $\mathrm{Na}$ in the ground " $\mathrm{S}$ " state - excited atom of $\mathrm{Na}$ in the "P" state is studied. In fig.1 we present the results of calculation for ionization cross section $\sigma_{i}^{(d i f)}$ as a function of the collision energy $E_{c}$ (in atomic units) under different values of effective quantum number $n_{o}^{*}$. For comparison there are also presented the results of calculation within the analytical Weisskopf model and numeral diffuse Weisskopf model [22]. In figure 2 it is presented a dependence of constant $K_{i}\left(\mathrm{~cm}^{-3}\right)$ of the velocity for entire ionization upon effective quantum number $n_{o}^{*}$ for reaction of collision in the crossing beams for pair " $N a(n P)+N(3 S)$ " under temperature of effusion sources $\mathrm{T}=600 \mathrm{~K}$. For comparison there are also presented the results of calculation on the basis of the Mihajlov-Janev model (1), analytical Weisskopf model (2), numeral diffuse Weisskopf model (3) and present approach (4); the squires denote the experimental data of Wang-Weiner (c.f.[18,22]). Results of present calculation are in sufficiently good agreement with experimental data of Wang-Weiner. But the most important conclusion of carried out calculation is in the increasing of the ionization cross section due to the realization of the stochastic drift of the Rydberg electron. Naturally, it is of a great importance a sufficiently long time of the elementary collision act for developing the stochastic instability. Further we consider the procedure of optimization of the laser photoionization sensor scheme.

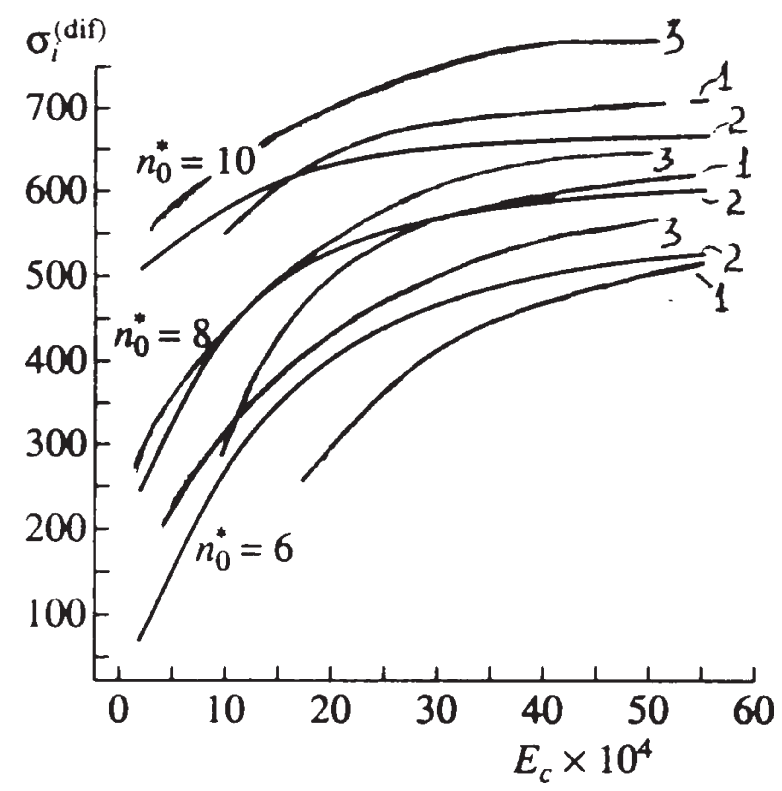

Fig.1. Collisional ionization cross section $\sigma_{i}^{(d i f)}$ as a function of the collision energy $E_{c}$ (in atomic units) under $n_{o}^{*}=6,8,10$ : (1) Analytical Weisskopf model; (2) Numerical diffuse Weisskopf model; (3) Present paper;

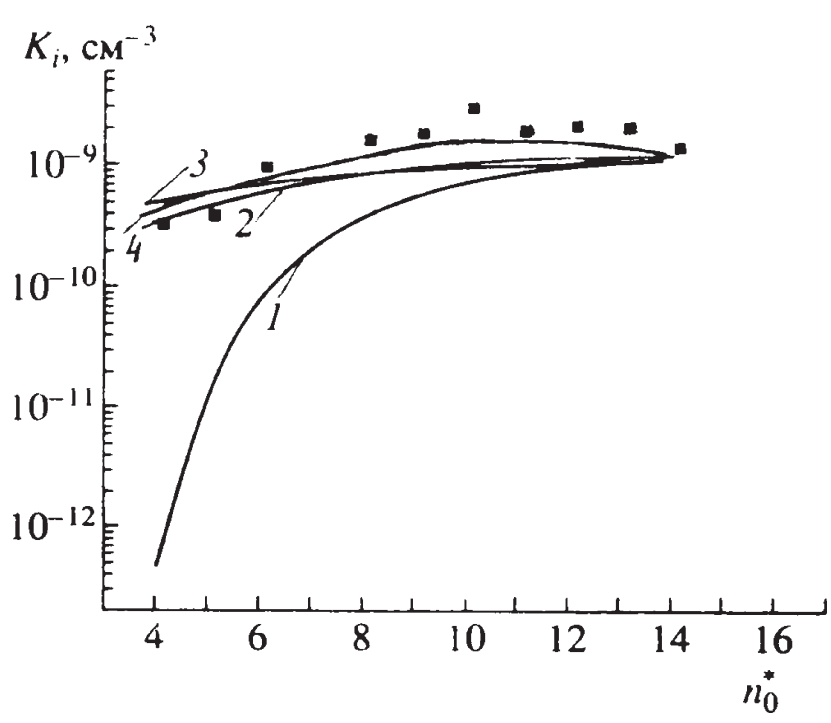

Fig.2. Dependence of constant $K_{i}\left(\mathrm{~cm}^{-3}\right)$ of the entire ionization velocity upon quantum number $n_{o}^{*}$ for reaction of collision in the crossing beams for pair" $\mathrm{Na}(n \mathrm{P})+N(3 S)$ " under temperature of effusion sources T=600 K: (1)- the Mihajlov-Janev model ; (2) Analytical Weisskopf model; (3) Numerical diffuse Weisskopf model; (4) Present approach;

\section{Optimization of the laser photoionization scheme}

The optimization procedure of the laser photoionization scheme is in a searching the optimal form of electromagnetic pulse of resonant radiation in order to provide a maximum of excited particles in the gases separation scheme (naturally this is one of the possible versions). This problem was in details considered in ref. [7-10]. It is important that in difference from the simplified model of speed equations (c.f.[1]) the separation process in our papers is described by more adequate density matrice equations system (c.f. $[12,13])$. As an example, we consider a scheme for the laser separation and sensing $\mathrm{Na}, U, C f$ isotopes. In experiment of Letokhov et al (c.f. [1]) two dye-lasers, pumped by one pulsed $N_{2}$ laser are used. The first laser parameters provided a saturation of the resonant transition: $3{ }^{2} S_{1 / 2} \rightarrow 3{ }^{2} P_{1 / 2}$ $\mathrm{Na}$. The wavelength of second laser is tuned in re-

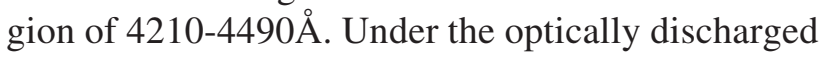
separation the scheme will be optimal if an atom is excited by laser radiation till state which has the decay probability due to the collisional ionization bigger than the radiation decay probability. In figure 3 we present the numeric modelling results of the optimal form of laser pulse in the photoionization 
scheme with stochastic collisional ionization. The data are obtained for the $\mathrm{Na}$ isotopes separation. It is also shown the typical behavior of the ground (curve 1) and excited (curve 2) states population. Note that the results presented are in qualitative aspect similar to analogous data with ionization by pulsed electric field and ionization by electron impact scenarios [8-10]. Let us remember data regarding the excitation and the ionization cross sections for considered system: the excitation cross section at the first step of the photoionization scheme is 10 ${ }^{12} \mathrm{~cm}^{2}$; the ionization cross-section from excited $3^{2} P_{2}$ state: $\sigma_{2}=10^{-17} \mathrm{~cm}^{2}$, from ground state $\sigma_{2}=10^{-19} \mathrm{~cm}^{2}$ [1]. One can see that the relation of these cross sections is $10^{5}$ and $10^{7}$ correspondingly. This fact provides the obvious non-efficiency of standard photoionization scheme. A use of the electric filed pulse at the second step for ionization of the excited atoms provides more optimal scheme from energetic point of view. For example, for the $3 p^{2} P_{1 / 2}-15^{2} D_{3 / 2}$ transition the corresponding cross section $7 \cdot 10^{-15} \mathrm{~cm}^{2}$ under strength of electric field $12 \mathrm{kV} / \mathrm{cm}$ [1]. Our calculation shows that the stochastic ionization cross section is $4,8 \cdot 10^{-14} \mathrm{~cm}^{2}$. So, from energetic point of view, the stochastic collisional ionization mechanism can be very perspective alternative to even electric field ionization mechanism. The similar data are obtained for the $\mathrm{U}$ isotopes separation. The laser photoionization scheme for $\mathrm{U}$ isotopes included the following steps: i). Laser excitement of the ${ }^{235} \mathrm{U}$ isotopes from the ground $5 \mathrm{f}^{3} 6 \mathrm{~d} \mathrm{~s}^{2}-5 \mathrm{~L}_{6}{ }^{0}$ state and low lying metastable $5 \mathrm{f}^{3} 6 \mathrm{~d} 7 \mathrm{~s}^{2}-{ }^{5} \mathrm{~K}_{5}{ }^{0}$ state with energy $620,32 \mathrm{~cm}^{-1}$; ii). Transition into the autoionization state with doubly excited external shell and then ionization by laser field or collision method. A scheme for sensing the nuclear reaction products is considered on example of the spontaneous fusion ${ }^{252} \mathrm{Cf}$ isotope on the unsymmetrical coils (in particular, Cs). Laser photoionization sensing the Cs isotopes was based on the resonant excitement of Cs (the following wavelengths are used: $6^{2} S_{1 / 2}-7^{2} P_{3 / 2}, 4555 \AA$ and $6^{2} S_{1 / 2}-7^{2} P_{1 / 2}, 4593 \AA$ ) and further ionization. So, in our case sensing the nuclear reaction products is resulted in detecting the $\mathrm{Cs}$ atoms as spontaneous nuclear fusion coils. A signal, which is determined by special surface-barrier nuclear detector, indicates on appearance of the single fusion of ${ }^{252} \mathrm{Cf}$ nucleus. Our data for Cs isotopes are qualitatively similar to data for $\mathrm{Na}, \mathrm{U}$ atoms. We believe that creation of the laser photoionization sensor on the basis of considered scheme would be more effective and perspective than in a case of other separating and sensing schemes.

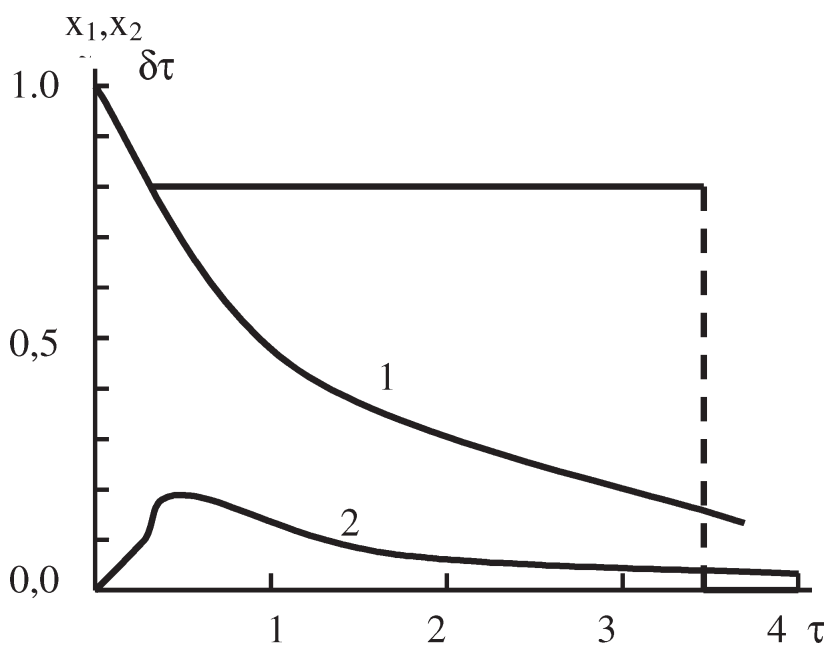

Fig.3. Results of numeric modelling for the optimal form of laser pulse for laser photoionization scheme of the $N a$ isotopes: d+dotted line - optimal form for laser pulse; curves 1 and 2 - the populations of the ground and excited states.

In conclusion let us formulate the key results of present paper. We proposed and studied new optimal scheme for the laser photoionization sensing the separating isotopes and nuclear reactions products, which is based on the selective laser excitation of atoms into excited Rydberg states and further stochastic collisional ionization. The operator perturbation theory, the Focker-Plank stochastic equation method, optimal laser action model and density matrices formalism are used for numerical modelling optimal scheme parameters for the $N a, U, C s(C f)$ isotopes detecting. At the first step of ionization scheme the $\delta$-pulse provides a maximally possible level of excitation for the up state. Then the stochastic collisional ionization must be realized earlier than the parasitic spontaneous relaxation processes (resonant re-charging etc. [1]) begin to destroy and change an achieved excitement level of atoms. It is important to note that the stochastic scenario is realized better when an elementary collisional act is sufficiently long for developing the stochastic instability. As a result, an additional condition for the potential photoionization scheme realization is connected with non-high temperature regime. In any case a stochastic scenario provides one of the possibilities for significant changing the output of charged particles due to the change of effective value for the reaction energy threshold.

Acknowledgement. Author is very thankful to Prof. A.V. Glushkov for useful comments and reading the manuscript. 


\section{References}

1. Letokhov V.S. Nonlinear Selective Photoprocesses in atoms and molecules. - M.,1983. - 408c.

2. Karlov N.V., Orlov A.I., Petrov Yu.N., Prokhorov A.M. Laser Governing by the diffusion stream of gases into vacuum through obstacle with developed surface// Izv. AS USSR.Ser.Phys. 1985. - Vol.49,N4. - P.500-505.

3. Stoll W. Present Status of industrial Isotope separation by laser technology// Atomic and Molecular Pulsed Lasers. - Tomsk: SO RAN, 2001. - P.71.

4. Buchanov V.V., Kazaryan M.A., Kalugin M.M., Prokhorov A.M. Laser separation of Silicon Isotopes by the AVLIS Technology// Atomic and Molecular Pulsed Lasers. - Tomsk: SO RAN, 2001. - P.72.

5. Glushkov A.V., Ambrosov S.V., Shpinareva I.M. Non-linear Selective Photoprocesses in Atoms and Molecules and their Optimal Governing. Optimized Isotope Separation Schemes// Atomic and Molecular Pulsed Lasers. — Tomsk: SO RAN, 2001. - P.70.

6. Glushkov A.V., Malinovskaya S.V., Ambrosov S.V., Shpinareva I.M. and Troitskaya O.V. Resonances in Quantum Systems in Strong External Fields: Consistent Quantum Approach// J. Techn. Phys. — 1997. — Vol.38,N2. - P.215-219.

7. Ambrosov S.V., Glushkov A.V. Atom in a DC electric field: Relativistic Operator Perturbation Theory Method// Proc. European Science Foundation REHE School and Workshop on "Spin-Orbit Coupling in Chemical Reactions". - Torun (Poland). - 1998. - P.23.

8. Ambrosov S.V., Shpinareva I.M. Selective Ionization of Atoms by Electric and Light Field. Autoionization Rydberg Resonances in Heavy Atoms. Optimal Isotope-Separation Selective Molecular Vibration Levels Excitation Schemes// Proc. 32 ${ }^{\text {nd }}$ Europhysics Conference EGAS. - Vilnius (Lithuania). - 2000. - P.197.

9. Glushkov A.V., Ambrosov S.V.,Ignatenko V.M., Non-hydrogenic atoms and Wannier-Mott exciton in a dc electric field: photoionization, stark effect, resonances in the ionization continuum and stochasticity / / Photoelectronics. - 2001. - Vol.10. - P.101-106.

10. Ambrosov S.V. Selective Photoionization of atoms: resonant re-charging in vapours of alkali elements and uranium // Photoelectronics. - 2002. Vol. 11. - P.40-45.

11. Ambrosov S.V., Glushkov A.V., DC Stark Effect in Non-hydrogen atoms: Operator Perturbation theory// Internat. Journ. Quantum Chem. - 2004. - Vol.98. - P.685-592.

12. Ivanov L.N., Letokhov V.S. Spectroscopy of autoionization resonances in heavy elements atoms// Com.Mod.Phys.D.:At.Mol.Phys. - 1985. Vol.4. - P.169-184.
13. Glushkov A.V., Ivanov L.N. Radiation Decay of Atomic States: atomic residue and gauge non-invariant contributions // Phys. Lett.A. - 1992. Vol.170,N1. - P.33-37.

14. Glushkov A.V.,Ivanov L.N. DC Strong-Field Stark-Effect: consistent quantum-mechanical approach// J.Phys.B: At. Mol. Opt. Phys. — 1993. Vol.26,N16. - P.L379-L386.

15. Ivanov L.N., Ivanova E.P., Knight L. Energy Approach to consistent QED theory for calculation of electron-collision strengths//Phys.Rev.A. - 1993. — Vol.48,N6. - P.4365-4374.

16.Glushkov A.V., Rusov V.D., Ambrosov S.V., Loboda A.V., Resonance states of compound super-heavy nucleus and EPPP in heavy nucleus collisions // New Projects and New Lines of research in Nuclear physics.Eds. Fazio G. and Hanappe F. - Singapore : World Scientific, 2003. - P.126-132.

17. Glushkov A.V., Ambrosov S.V., Loboda A.V., Khetselius O.Y., Svinarenko A.A. QED calculation of the superheavy elements ions: energy levels, radiative corrections and hyperfine structure for different nuclear models// Nucl. Phys.A.: Nucl.and Hadr. Phys. -2004. — Vol. 708. - P.513-518.

18. Photonic, Electronic, Atomic Collisions. - Singapore: World Sc.. — 1997. — P.621-630.

19. Taylor G. Theory of scattering. Quantum theory of non relativistic collisions. - Moscow, Mir, 1991. $-565 \mathrm{p}$.

20. Smirnov B.M. Ionization processes under slow atomic collisions// Usp.fys.Nauk. — 1981. Vol.133. - p.569-613.

21. Manakov N.L., Ovsyannikov V.D., Ostrovsky V.N., Yastrebov V.N. Influence of longacting forces on the Peninng-ionization// Opt. Spectr. 1984. - Vol.56,-P.222-226.

22. Bezuglov N.N., Borodin B.M., Kazansky A.K. et al, Analysis of stochastic equations of the FockerPlank with valuable boundary conditions in elementary process of collisional ionization//Opt. Spectr.-2001. - Vol.89. - P.25-33.

23. Glushkov A.V., Ambrosov S.V., Gopchenko E.D. et al, Calculation of spectroscopic characteristics for wan-der-vaalse olecules. Atom of inert gasatom of galogen in ground state// Opt. Spectr. 1998. - Vol.84. - P.567-570.

24. Glushkov A.V., Ambrosov S.V., Serbov N.G. et al, Calculation of oscillator strengths in Na-,Fr-like multi-charged ions// Journ. Appl.Spectr. - 1996. - Vol.63,N1. - P.39-45.

25. Glushkov A.V., Ambrosov S.V., Prepelitsa G.P., Auger effect in atoms and solids: Calculation of characteristics of the Auger decay in atoms, quasimolecules and solids with application to surface composition analysis// Functional Materials. 2003. - V.4. - P.217-222. 